\title{
Dyscalculia: Characteristics, Causes, and Treatments
}

\author{
Gavin R. Price \\ gavinprice@gmail.com \\ Daniel Ansari \\ Western University, daniel.ansari@gmail.com
}

Follow this and additional works at: https://digitalcommons.usf.edu/numeracy

Part of the Disability and Equity in Education Commons, Psychological Phenomena and Processes Commons, and the Special Education and Teaching Commons

\section{Recommended Citation}

Price, Gavin R., and Daniel Ansari. "Dyscalculia: Characteristics, Causes, and Treatments." Numeracy 6, Iss. 1 (2013): Article 2. DOI: http://dx.doi.org/10.5038/1936-4660.6.1.2 


\title{
Dyscalculia: Characteristics, Causes, and Treatments
}

\begin{abstract}
Developmental Dyscalculia (DD) is a learning disorder affecting the ability to acquire school-level arithmetic skills, affecting approximately $3-6 \%$ of individuals. Progress in understanding the root causes of DD and how best to treat it have been impeded by lack of widespread research and variation in characterizations of the disorder across studies. However, recent years have witnessed significant growth in the field, and a growing body of behavioral and neuroimaging evidence now points to an underlying deficit in the representation and processing of numerical magnitude information as a potential core deficit in DD. An additional product of the recent progress in understanding DD is the resurgence of a distinction between 'primary' and 'secondary' developmental dyscalculia. The first appears related to impaired development of brain mechanisms for processing numerical magnitude information, while the latter refers to mathematical deficits stemming from external factors such as poor teaching, low socioeconomic status, and behavioral attention problems or domain-general cognitive deficits. Increased awareness of this distinction going forward, in combination with longitudinal empirical research, offers great potential for deepening our understanding of the disorder and developing effective educational interventions.
\end{abstract}

\section{Keywords}

Dyscalculia, numeracy, quantitative reasoning, cognition, number sense

\section{Creative Commons License}

$$
\text { c) (i) (8) }
$$

This work is licensed under a Creative Commons Attribution-Noncommercial 4.0 License

\section{Cover Page Footnote}

Gavin Price is an Assistant Professor in Educational Neuroscience in the Department of Psychology and Human Development at Vanderbilt University, Peabody College. He completed his PhD in Developmental Cognitive Neuroscience at the University of Jyvaskyla, Finland, under the Marie Curie Research Training Network 'NUMBRA' (Numeracy \& Brain Development) in 2008 and then completed a postdoctoral fellowship at the University of Western Ontario under the supervision of Daniel Ansari.

Daniel Ansari is Canada Research Chair in Developmental Cognitive Neurosicence in the Department of Psychology at the University of Western Ontario, Canada, where he is the principal investigator in the 'Numerical Cognition Laboratory' (www.numericalcognition.org) and also a member of the Institute for Brain and Mind. He completed his PhD at University College London, UK, in 2003. From 2003-2006 he was an Assistant Professor in the Department of Education at Dartmouth College. 


\section{Introduction}

Today's world requires us to process unprecedented levels of numerical information. Computers, smartphones, financial and healthcare information processing are just a few of the many contemporary demands requiring our numerical fluency. Despite this landscape, up to $25 \%$ of "economically active" individuals in countries such as the United Kingdom remain "functionally innumerate" (Gross, Hudson, and Price 2009); for those individuals, rates of unemployment, mental and physical illness, arrest and incarceration are higher (Duncan et al. 2007; Parsons and Bynner 2005; Bynner and Parsons 1997). At the societal level, low numeracy has been estimated to cost the UK government up to $£ 2.7$ billion in lost revenues and added costs, and widespread improvements in mathematical competence have been linked to observable increases in the gross domestic product (GDP) (OECD 2010). Thus it is essential, for continued development of effective quantitative learning and mathematical education methods, that we understand the sources of such widespread and debilitating numerical and mathematical impairments.

While many factors such as educational experience, IQ and other cognitive abilities, and motivation may undermine the development of numeracy skills, one key potential impediment is a developmental learning disorder that is specific to numeracy. Developmental Dyscalculia (DD) is such a learning disorder that specifically affects the ability to acquire school-level arithmetic skills. Diagnosis of DD is recommended by the DSM-IV ${ }^{2}$ when "mathematical ability, as measured by individually administered standardized tests, is substantially below that expected given the person's chronological age, measured intelligence, and age-appropriate education."

Studies on representative samples of both school-based and general populations have been carried out in various countries across the world, and the resulting estimates suggest that as many as 3-6\% of individuals may suffer from DD (for a review see Shalev et al. 2000). Such prevalence estimates suggest that a high number of functionally innumerate individuals may be so because they suffer from a specific learning disorder, akin to Dyslexia ${ }^{3}$ in the case of reading. Therefore, from the perspective of educators, those individuals may require tailored educational interventions to improve their numeracy skills. Such interventions can be tailored to individuals only on the basis of improved understanding of the causes and characteristics of the disorder itself.

\footnotetext{
${ }^{1}$ lacking the "essential knowledge, skills, and understanding that will enable them to operate confidently effectively and independently in life and at work" (DfES 2005).

${ }^{2}$ The 'DSM' is the 'Diagnostic and Statistical Manual of Mental Disorders' published by the American Psychiatric Association. DSM-IV refers to the $4^{\text {th }}$ iteration of this publication.

${ }^{3}$ A developmental learning disorder specifically affecting the acquisition of fluent reading skills.
} 
Despite the evident importance of numerical and mathematical skills for life success and a prevalence rate equivalent to that of developmental dyslexia (Shalev et al. 2000), DD has been chronically understudied, with studies on dyslexia outnumbering those on DD by 14:1 as recently as 2007 (Gersten, Clarke, and Mazzocco 2007). The consequence of this under-attention is that the cognitive causes of DD are currently poorly understood. It should be noted that DD often co-occurs (is comorbid) with other learning difficulties such as Developmental Dyslexia and Attention Deficit Hyperactivity Disorder (ADHD). ${ }^{4}$ However, the focus of the present review is on DD alone rather than on cases in which DD and other disorders co-occur.

Research into DD has revealed a wide range of behavioral deficits in mathematical abilities. However, the consistent identification of a core group of behavioral markers across studies has remained elusive. This general lack of consistency can be attributed to two primary factors, aside from the relative lack of attention paid to DD. First, variation across studies in criteria used to identify children with mathematical difficulties has impeded the achievement of a consensus on the defining features of DD. To elaborate, some studies have employed discrepancy criteria, such as defining DD as math performance within an affected individual as equivalent to children one or two years younger (Temple and Sherwood 2002; Shalev, Manor, and Gross-Tsur 1997). Other studies have used percentile cut-off points, ranging from the lowest $35^{\text {th }}$ percentile (Geary, Hamson, and Hoard 2000) to the lowest $11^{\text {th }}$ percentile (Butterworth 2003). Still more studies have employed alternatives to discrepancy criteria, such as a standard deviation criterion in which a child is deemed dyscalculic if the child scored three standard deviations below the mean on item-timed arithmetic for example (Landerl, Bevan, and Butterworth 2004). Such wide-ranging selection criteria have the effect of including individuals whose math deficits do not stem from a persistent learning disorder, but rather may stem from exogenous sources such as poor teaching, low socio-economic status, or alternative developmental disorders such as ADHD. This fact underlines then the second reason for the lack of consensus on the behavioral profile of DD: mathematical skills are inherently heterogeneous and, as such, are vulnerable to disruption from a wide range of endogenous and exogenous sources.

In 1970, Ladislav Kosc proposed a definition of DD:

Developmental dyscalculia is a structural disorder of mathematical abilities which has its origin in a genetic or congenital disorder of those parts of the brain that are the direct anatomico-physiological substrate of

\footnotetext{
4 A mental or neurobehavioral disorder characterized by either significant difficulties of inattention or hyperactivity and impulsiveness or a combination of the two (National Institute of Neurological Disorders and Stroke. National Institute of Health)
} 
the maturation of mathematical abilities adequate to age, without a simultaneous disorder of general mental functions (Kosc, 1970, p. 192).

In that definition is an inherent distinction between what Kosc terms primary dyscalculia, namely, math deficits stemming from an impaired ability to acquire those skills, versus secondary dyscalculia (or "pseudo-dyscalculia"), namely, math deficits caused by external factors such as those mentioned above. In line with Kosc's proposed taxonomy, recent years have seen a growing number of researchers (e.g., Rubinsten and Henik, 2009) distinguishing between pure DD as an endogenous learning disorder and mathematical learning disabilities/ difficulties (MLD), driven by exogenous factors or cognitive deficits not specific to numerical processing, such as working-memory, visual-spatial processing or attention. Attention to this distinction is beginning to reveal distinct pathological profiles, whereby children with the most-severe math deficits exhibit cognitive deficits in very basic number processing which tap "the number sense," while children with more moderate impairments do not (Mazzocco, Feigenson, and Halberda 2011). Thus it may be that the primary vs. secondary distinction in DD is somewhat analogous to a distinction between the severity of presented math difficulties.

The following review will summarize the current state of knowledge regarding DD, drawing from a range (but not exhaustive list) of empirical studies, many of which likely include in their samples individuals with both primary and secondary DD. In so doing, we outline the defining behavioral and brain-level characteristics of primary $\mathrm{DD}$, and highlight the importance of distinguishing between primary and secondary DD in future research.

\section{Behavioral Characteristics}

Traditionally, the defining features of DD have been poor retrieval of arithmetic facts from memory and the perseverant use of immature calculation strategies (Geary and Hoard 2005). However, a growing body of behavioral and neuroimaging evidence, emerging over the last decade, suggests that DD may be rooted in impairments of a neurobiological system for processing numerical magnitudes (the total number of items in a set) and that it is this impairment that, over the course of learning and development, gives rise to the difficulties in the retrieval of arithmetic facts. Debate still exists, however, as to the role of domaingeneral cognitive factors, such as working-memory and spatial attention, in the etiology of DD.

\section{Arithmetic}

The most consistently observed behavioral hallmark of DD is impaired arithmetic fact retrieval (Mazzocco, Devlin, and McKenney 2008). As early as grades 1 and 
2, typically developing children undergo a developmental shift in their calculation strategies. They begin by solving simple problems through procedural methods such as counting, but usually by $3^{\text {rd }}$ grade, they have developed a store of arithmetic facts in memory, from which they can quickly recall the solution to a given problem (Ashcraft 1982). Children with DD, on the other hand, typically fail to develop such fluent fact-retrieval mechanisms, continuing to employ procedural strategies long after their typically developing peers have progressed to memory-based strategies (Geary 1993; Geary, Bow-Thomas, and Yao 1992; Geary, Hamson, and Hoard 2000; Jordan and Hanich 2003; Hanich et al. 2001; Landerl, Bevan, and Butterworth 2004; Russell and Ginsburg 1984). As an indicator of the severity of the fact-retrieval deficit in DD children, typically developing children have been found to recall an average of three times as many arithmetic facts as those with DD (Hasselbring et al. 1988).

A corollary of impaired fact retrieval in DD is the use of immature or inefficient problem-solving strategies. If a child with DD has not learned a given arithmetic fact, and hence cannot recall it fluently from memory, he/she will resort to procedural strategies, which are often sub-optimal and overly laborious. For example, children with DD in the first and second grades frequently adopt a count all method to solve simple calculations, whereby the child starts with zero and counts both addends until the solution is reached. By contrast, typically developing children of the same age might employ a count min strategy, starting with the larger addend and counting from there (Geary, Hamson, and Hoard 2000; Geary, Hoard, and Hamson 1999).

One of the difficulties in reliably characterizing the behavioral profile of DD is that math difficulties may emerge at varying stages of the educational process. For example, Mazzocco and Myers (2003) reported that, $65 \%$ of a sample of DD children in grade 3 had met the diagnosis criteria for DD in kindergarten, whereas $20 \%$ of the sample had first met the criteria upon reaching grade 2 . That finding underlines the fact that the skills required for successful mathematical performance change over the course of development, and thus some children may have a specific deficit at an early learning stage, which then disrupts the acquisition of later skills. This disruption may occur because the foundational skills are simply not present, or because inefficient or immature procedural skills result in extra effort required to carry out simple calculations. This extra effort spent on elementary skills in turn renders children unable to follow and learn more complex procedural knowledge being taught in the classroom (Pellegrino and Goldman 1987). The finding of changing profiles over time also highlights the importance of looking for persistent deficits in the diagnosis of DD. In other words, it is important that children show deficits in math performance at more than one time point. 
While deficits in arithmetic fact retrieval and strategy use define the phenotypic expression of DD at the school-level, they are also exhibited by individuals with secondary DD/MLD. Thus when retrieval deficits are used as the sole definitional criterion for DD it may complicate identification of the root cause of the disorder. Several researchers have suggested that primary DD might be driven by a core deficit of "the number sense," a cognitive mechanism that supports the representation and processing of numerical magnitudes (Butterworth 1999; Dehaene 1997). Accordingly, a large proportion of recent research has investigated the function of that number sense in children with DD.

\section{Basic Number Processing}

In one of the first studies to examine basic numerical processing in children with mathematical learning difficulties, Koontz and Berch (1996) reported that atypically developing children do not show the same interference from numerical information when judging whether two numbers presented in different formats are identical or not. This suggests that numerical magnitude information is not activated automatically in DD children as it is in their typically developing peers. The suggestion of reduced automatic activation of semantic numerical representations in DD was subsequently supported by Rubinsten and Henik (2005), who reported a lack of facilitation from numerical information in DD children during a numerical stroop task. ${ }^{5}$ A lack of automaticity in processing numerical information does not itself indicate whether the underlying semantic representation is impaired, or whether there is a deficit in the link between the semantic representations and their symbolic referents (i.e., Arabic digits). Thus, many researchers have employed the numerical comparison paradigm ${ }^{6}$ as a method of probing the integrity of numerical magnitude representations.

Early reports from case studies (Butterworth 1999), as well as studies using a wide range of math difficulties (i.e., $30^{\text {th }}$ percentile) (Geary, Hoard, and Hamson 1999) suggested impaired performance in DD individuals during numerical comparison. Subsequent studies using more-stringent selection criteria confirmed deficient number-comparison skills in DD children, and have even shown impaired basic number naming (Landerl, Bevan, and Butterworth 2004; van der Sluis, de Jong, and van der Leij 2004), suggesting the presence of very elementary deficits in basic number processing in DD. Importantly, DD children not only show increases in global reaction time and error rate during number comparison, but also a qualitatively different "distance effect" (Mussolin. Mejias et al. 2010).

\footnotetext{
${ }^{5}$ The numerical stroop task requires participants to select which of two simultaneously presented Arabic digits is physically larger. Which of the two numbers is physically larger can be either congruent or incongruent with with which of the numbers is numerically larger

${ }^{6}$ A task that requires participants to select which of two numbers is numerically larger.
} 
The distance effect (Moyer and Landauer 1967) refers to the behavioral phenomenon that, as the distance between two numbers being compared decreases (e.g., 2 - 9 versus 7 -9), reaction times and errors increase. In other words, numbers that are closer together are harder to compare than numbers that are further apart. The numerical distance effect (NDE) is taken by many researchers to reflect the integrity of the underlying representation of numerical magnitude along a "mental number line" (Dehaene 2003), with a larger NDE indicating a less-precise or more noisy representation.

In support of this idea, the NDE decreases in size over the course of development (Sekuler and Mierkiewicz 1977), suggesting an ontogenetic increase in the precision of the number sense. Children with DD have been shown to have larger NDEs than typically developing children (Ashkenazi, Mark-Zigdon, and Henik 2009; Price et al. 2007), in much the same way that typically developing children show a larger NDE relative to adults, suggesting that DD children may have a less-refined, immature representation of numerical magnitude compared to their typically developing peers. Recent evidence suggests that the magnitude of the developmental delay in the precision of this representation may be on the order of five years, with DD children showing numerical-representation precision equivalent to typically developing children five years their junior (Piazza et al. 2010).

It appears, therefore, as though DD, defined by impaired arithmetic skills, is associated with deficient basic numerical magnitude processing, pointing to a developmental impairment or delay in the number sense as a possible root cause. However, recent evidence suggests that only the most severely impaired DD children (10\% percentile) show impaired acuity of numerical magnitude representations, while those with below average math skills $\left(11^{\text {th }}-25^{\text {th }}\right.$ percentile $)$ do not differ from typically developing children (Mazzocco, Feigenson, and Halberda 2011). This finding suggests that primary DD may be associated with more-severe arithmetic deficits, and attributed to a congenital impairment of the ability to represent and process numerical magnitude information, falling nicely in line with Kosc's early definition (see above). Secondary DD, on the other hand, may be associated with less-severe arithmetic difficulties that are not related to impaired numerical magnitude representation and processing. Thus, the need to differentiate between primary and secondary DD becomes abundantly clear when considering differences in a) their etiology and b) their phenotypic severity.

\section{Non-numerical Deficits}

Despite the generally accepted definition of DD as a learning disorder specific to arithmetic, several researchers suggest that its root cause may lie in disturbances 
of domain-general cognitive mechanisms such as working memory, visual-spatial processing, or attention.

In support of this hypothesis, several studies have shown that children with mathematical difficulties underperform on tests of various aspects of working memory, such as the phonological loop ${ }^{7}$ (Hitch and McAuley 1991; Koontz and Berch 1996; McLean and Hitch 1999), visuo-spatial sketch pad ${ }^{8}$ (McLean and Hitch 1999), and forward and backward digit-span ${ }^{9}$ (Geary, Brown, and Samaranayake 1991; Geary, Hoard, and Hamson 1999; Passolunghi and Siegel 2004). These findings could be taken to indicate a tight coupling between deficits of working memory and arithmetic learning difficulties; however, many of the above studies used selection criteria (e.g., $30^{\text {th }}$ percentile) broad enough to make it likely that their samples included children with primary and children with secondary DD. In contrast, several studies with more-stringent selection criteria (e.g., three standard deviations below the mean on item-timed arithmetic) found no differences between DD and typically developing children on working memory measures (Landerl, Bevan, and Butterworth 2004; Temple and Sherwood 2002). While it is clearly understood that working memory is an essential cognitive component for the acquisition of arithmetic skills, the existing literature suggest that those individuals with both working memory problems and arithmetic deficits may be best categorized as suffering from secondary DD. Meanwhile primary DD, the more-severe disorder, appears to be relatively independent of working memory impairments.

Deficits in visuo-spatial attention have also been put forth as a possible domain-general cause of DD (e.g., Geary 2004), due to the important role of visuo-spatial processing in arithmetic processing. Several studies have reported poorer performance on tests of attention and visual-spatial processing in DD children relative to controls (e.g., Shalev, Auerbach, and Gross-Tsur, 1995; Lindsay, Tomazic, Levine, and Accardo, 2001). Furthermore, Ashkenazi, Rubinsten, and Henik (2009) argue that the lack of facilitation in DD children in numerical stroop tasks is driven by difficulty in recruiting attention, rather than impaired numerical magnitude representations. In support of this, they report that individuals with pure DD show deficient performance on tests of executive function and attentional alertness relative to controls. These findings suggest that individuals with DD may indeed present with atypical visual attention profiles;

\footnotetext{
${ }^{7}$ The component of Baddeley and Hitch's working memory model that processes and encodes auditory information (Baddeley and Hitch 1974).

${ }^{8}$ The component of Baddeley and Hitch's working memory model that processes and encodes visual information(Baddeley and Hitch 1974).

${ }^{9}$ Participants are presented with a series of digits (e.g., " $8,3,4$ ") and must immediately repeat them back, either in the order presented or in reverse order.
} 
however, recent evidence using physical vs. numerical line-bisection tasks ${ }^{10}$ suggests that deficits in spatial and numerical processing in DD may in fact be dissociable (Ashkenazi and Henik 2010). Further research is required to disentangle the role of visual-spatial attention in DD.

Finally, some researchers have suggested that the root cause of DD may lie in a disruption of the mapping between Arabic digits and their numerical magnitudes (Rousselle and Noel 2007). This "access deficit hypothesis" stems from evidence showing a specific impairment in symbolic (e.g., Arabic numerals) but not nonsymbolic (e.g., dot arrays) numerical comparison, taken to indicate intact underlying representations of numerical magnitude (Rousselle and Noel 2007). To date, it is unclear whether the numerical magnitude processing deficits exhibited by children with DD are irrespective of the format in which the numbers are presented or whether the deficits emerge when children acquire the meaning of numerical symbols.

\section{Neural Characteristics}

If primary DD exists as a specific, endogenously driven learning disorder in the way that Kosc (1970) suggested, then it is necessary to demonstrate in individuals with primary DD "a genetic or congenital disorder of those parts of the brain that are the direct anatomico-physiological substrate of the maturation of mathematical abilities...."

Paying particular attention to the idea of an impairment of the neural mechanisms supporting "the maturation" of math skill, then, the behavioral evidence discussed above suggests the most likely deficit would be in the neural substrates of numerical magnitude processing. Neuroimaging research in typically developing adults and children has identified the intraparietal sulcus (IPS, Fig. 1) as a key brain region involved in the processing of numerical magnitude representation (Dehaene et al. 2003; Cohen, Lammertyn, and Izard 2008). Thus, if primary DD is related to a core deficit

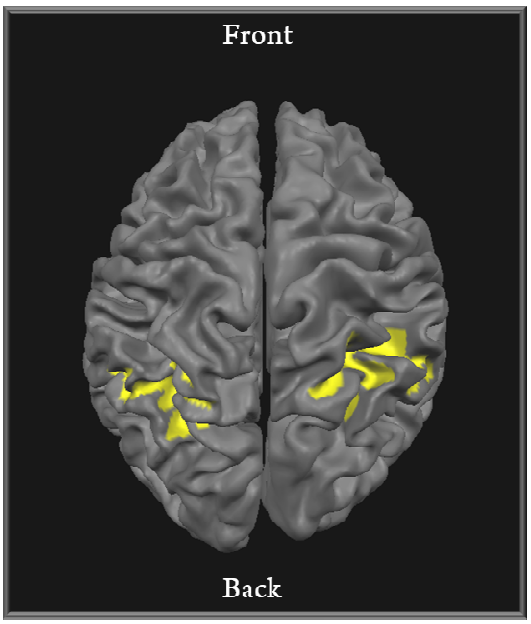

Figure 1. Three-dimensional rendering of an adult human brain. The left and right Intraparietal Sulci (IPS) are highlighted in yellow.

\footnotetext{
${ }^{10}$ Participants are required to indicate the center point of lines of different lengths.
} 
in "the number sense" evident at the brain level, then individuals with DD can be expected to show atypical activation of the IPS when processing numerical magnitude information. While only a handful of studies to date have tested this robustly, this hypothesis is gaining increasing levels of empirical support.

At the functional level, Price et al. (2007) reported reduced modulation of the right IPS in DD children during a nonsymbolic numerical comparison task (i.e., comparing which of two sets of squares was the more numerous). In that study, typically developing children showed greater activation in the IPS for number pairs that were closer together (small numerical distance) compared to pairs that were separated by a comparatively larger numerical distance. In contrast, DD children showed no such effect of numerical distance on brain activation. That study provided the first evidence of atypical brain activation during numerical magnitude processing independent of symbolic number use (i.e., comparison stimuli were nonsymbolic). Subsequent studies reported similar results using symbolic number comparisons (Arabic digits) (Mussolin, De Volder et al. 2010), suggesting a brain-level impairment in basic numerical magnitude processing in DD. It is important to note that in both studies, DD children were identified based on their arithmetic performance, yet they showed atypical brain activation during basic numerical magnitude processing. This is important to consider because it provides a clear link between the brain circuitry underlying numerical magnitude processing and arithmetic achievement. Furthermore, there is recent evidence that DD children show reduced activation of the IPS during mental arithmetic (Ashkenazi et al. 2012), suggesting that the developmental dysfunction of the IPS in DD children is associated not only with the foundational capacity of basic magnitude processing, but also with the phenotypic expression of impaired arithmetic skills.

It should be noted that some recent studies also have pointed to an overlap between the role of the IPS in numerical magnitude processing and spatial working memory. Rotzer et al. (2009) reported that DD children show less activation of the right IPS during a spatial working memory task (an adaptation of the corsi block-tapping task ${ }^{11}$ ) than control children. Consequently, the authors suggest that deficits in spatial working memory might "lie at the core of difficulties in non-symbolic numerical magnitude processing" (p. 2863). It is unclear, however, what the mechanistic link might be between spatial working memory and nonsymbolic magnitude processing, apart from a shared neural substrate. Thus, it is just as plausible to say that nonsymbolic magnitude processing deficits undermine the neural response during the corsi block-tapping task. Ultimately, both options are possible, but at present, the weight of evidence is in favor of a deficit in numerical magnitude processing.

${ }^{11}$ A task requiring mimicking a researcher as he/she taps a sequence of up to nine identical spatially separated blocks 
As well as showing atypical functional activation profiles, a growing body of evidence suggests that children with DD show atypical structural organization of the IPS. Using voxel-based morphometry, ${ }^{12}$ Rotzer et al. (2007) reported reduced grey matter volume in the right IPS of DD children relative to controls, while Rykhlevskaia et al. (2009) reported atypical white matter tracts linking the right IPS to the right fusiform gyrus (part of the ventral visual cortex).

Thus, it appears that DD is associated with atypical functional and structural characteristics of brain regions that support the processing of numerical magnitude information. It is possible, therefore, to speculate that the neurocognitive mechanisms for representing and processing numerical magnitude serve as a foundation for the acquisition of school-level arithmetic skills and that, in children with DD, the impairment of that foundation undermines the acquisition of those skills. However, what is currently absent is direct causal evidence of that relationship. To date, all neuroimaging studies of DD have been cross-sectional, making it impossible to know if atypical development of the IPS undermines the acquisition of math skills or vice-versa. Only future longitudinal work will be able to fully resolve this issue

\section{Treatment}

The inherent goal in refining our understanding of the behavioral and neural characteristics of DD is to inform the development of better educational interventions. Neuroscientific evidence can provide targeted direction for intervention approaches by identifying core neurocognitive mechanisms in need of remediation.

Two adaptive computerized training tools have been developed based on cognitive neuroscience evidence with the aim of remediating DD. The first, "The Number Race" is designed to improve the precision of numerical magnitude representations in DD (Wilson, Revkin, and Cohen 2006). The game asks children to select the larger of two arrays of dots and, in addition to providing feedback on the correct response, adjusts the numerical difference between the sets based on performance, making the task easier or more difficult. The second program, "Graphogame," follows a similar logic to The Number Race, requiring individuals to compare sets of objects. In contrast to The Number Race, which focuses on approximate estimation, Graphogame focuses on exact numerosities, ${ }^{13}$ and seeks to link those with number symbols (Arabic digits). While both programs target cognitive processes thought to be crucial for the development of math skills, and both result in improvements in number-comparison performance, neither program

\footnotetext{
${ }^{12}$ A method of analyzing neuroimaging data that allows the investigation of differences in the concentration of grey matter in specific regions of the brain.

13 'Numerosity' refers to the total number of items in a set.
} 
results in training effects that generalize to counting and arithmetic (Räsänen et al. 2009).

Other intervention programs, whose publishers reported use of neuroscientific evidence in their design, have also produced mixed results, either providing insufficient evidence to assess evaluation (e.g., "Fluency and Automaticity through Systematic Teaching with Technology: FASTT Math"), or demonstrating positive results limited to specific socio-economic demographics (e.g., "Numberworlds", Griffin 2007) (for a review see Kroeger, Brown, and O'Brien, 2012).

Thus, while still in the early days, attempts to directly train core cognitive mechanisms that are impaired in DD (i.e., numerical magnitude processing) do not appear to be delivering the effectiveness and transfer effects that would be hoped. Much future work is needed to understand the scaffolding relationship between foundational competencies and higher-level skills such as arithmetic, and how best to enhance that structured learning. With that in mind, it will be of key importance in developing effective educational interventions to strengthen our understanding of how basic processes and higher skills change, both in their nature and in relationship to each other, over the course of ontogenetic development.

\section{Conclusions}

Mathematical performance deficits, Developmental Dyscalculia, may arise because of a wide range of factors, from poor teaching, to low socio-economic status, to behavioral attention problems. However, a subset of children with math difficulties, possibly with the most-severe impairments, appears to suffer from a developmental learning disorder that undermines the ability to process basic numerical magnitude information, and that impairment in turn undermines the acquisition of school-level arithmetic skills. This disorder, "primary developmental dyscalculia," should not be confused with "secondary developmental dyscalculia," which refers to mathematical deficits stemming from external factors such as those described above. Instead, primary DD is associated with impaired development of brain mechanisms for processing numerical magnitude information and is thus driven by endogenous neurodevelopmental factors. While recent years have seen a growing body of evidence supporting the above characterization of primary DD, attempts to develop educational interventions on the basis of those findings have not proved successful. That said, it must be remembered that research in this area is in relative infancy when compared to research investigating developmental dyslexia, and thus, progress to date is exciting, with promises of rich future rewards. Key to maximizing the outcomes of this research is for future studies to focus on the causal relationship 
between numerical magnitude processing and later math skills, and on the role of development in the design of effective intervention tools.

\section{References}

Ashcraft, M. H. 1982. The development of mental arithmetic: A chronometric approach. Developmental Review 2 (3) (September): 213-236. http://dx.doi.org/10.1016/02732297(82)90012-0

Ashkenazi, S., and A. Henik. 2010. A disassociation between physical and mental number bisection in developmental dyscalculia. Neuropsychologia 48 (10) (August): 2861-8. http://dx.doi.org/10.1016/j.neuropsychologia.2010.05.028

Ashkenazi, S., N. Mark-Zigdon, and A. Henik. 2009. Numerical distance effect in developmental dyscalculia. Cognitive Development 24 (4): 387-400. http://dx.doi.org/10.1016/j.cogdev.2009.09.006

Ashkenazi, S., M. Rosenberg-Lee, C. Tenison, and V. Menon. 2012. Weak task-related modulation and stimulus representations during arithmetic problem solving in children with developmental dyscalculia. Developmental Cognitive Neuroscience 2 (February): S152-S166. http://dx.doi.org/10.1016/j.den.2011.09.006

Ashkenazi, S., O. Rubinsten, and A. Henik. 2009. Attention, automaticity, and developmental dyscalculia. Neuropsychology 23 (4): 535-540. http://dx.doi.org/10.1037/a0015347

Baddeley, A.D., and G. J. Hitch. 1974. Working memory. In The Psychology of Learning and Motivation, ed. G.A. Bower, 47-89, Academic Press

Butterworth, B. 1999. The Mathematical Brain. London: Macmillan.

- 2003. Dyscalculia Screener. London: NFER Nelson Publishing Company Ltd.

Bynner, J., and S. Parsons. 1997. Does numeracy matter? London: The Basic Skills Agency.

Cohen K. R., J. Lammertyn, and V. Izard. 2008. Are numbers special? An overview of chronometric, neuroimaging, developmental and comparative studies of magnitude representation. Progress in Neurobiology 84 (2): 132-147. http://dx.doi.org/10.1016/j.pneurobio.2007.11.001

Dehaene, S. 1997. The Number Sense. Oxford: Oxford University Press.

- 2003. The neural basis of the Weber-Fechner Law: A logarithmic mental number line. Trends in Cognitive Sciences 7 (4): 145-147. http://dx.doi.org/10.1016/S1364-6613(03)00055-X

- M. Piazza, P. Pinel, and L. Cohen. 2003. Three parietal circuits for number processing. Cognitive Neuropsychology 20 (3-6) (May 1): 487-506. http://dx.doi.org/10.1080/02643290244000239

DfES (Department for Education, UK). 2005. 14-19 Education and Skills: Implementation Plan.- Summary. https://www.education.gov.uk/publications/standard/publicationDetail/Page1/UOB \%202037\%202005\#downloadableparts (accessed Nov. 19, 2012)

Duncan, G. J., C. J. Dowsett, A. Claessens, K. Magnuson, A. Huston, P. Klebanov, L. S. Pagani, et al. 2007. School readiness and later achievement. Developmental 
Psychology 43 (6) (November): 1428-46. http://dx.doi.org/10.1037/00121649.43.6.1428

Geary, D. C. 1993. Mathematical disabilities: Cognitive, neuropsychological, and genetic components. Psychol Bull 114 (2) (September): 345-362. http://dx.doi.org/10.1037/0033-2909.114.2.345

_. 2004. Mathematics and learning disabilities. J Learn Disabil 37 (1): 4-15. http://dx.doi.org/10.1177/00222194040370010201

— and M. K. Hoard. 2005. Learning disabilities in arithmetic and mathematics. In Handbook of Mathematical Cognition, ed. J. I. D. Campbell, 253-267. New York: Psychology Press.

Geary, D. C, C. C. Bow-Thomas, and Y. Yao. 1992. Counting knowledge and skill in cognitive addition: A comparison of normal and mathematically disabled children. $J$ Exp Child Psychol 54 (3): 372-391. http://dx.doi.org/10.1016/0022-0965(92)90026$\underline{3}$

Geary, D. C., S. C. Brown, and V. A. Samaranayake. 1991. Cognitive addition: A short longitudinal study of strategy choice and speed-of-processing differences in normal and mathematically disabled children. Developmental Psychology 27 (5) (September): 787-797. http://dx.doi.org/10.1037/0012-1649.27.5.787

Geary, D. C., C. O. Hamson, and M. K. Hoard. 2000. Numerical and arithmetical cognition: A longitudinal study of process and concept deficits in children with learning disability. J Exp Child Psychol 77 (3) (November): 236-263. http://dx.doi.org/10.1006/jecp.2000.2561

Geary, D C, M. K. Hoard, and C. O. Hamson. 1999. Numerical and arithmetical cognition: Patterns of functions and deficits in children at risk for a mathematical disability. J Exp Child Psychol 74 (3) (November): 213-239. http://dx.doi.org/10.1006/jecp.1999.2515

Gersten, R., B. Clarke, and M. M. M. Mazzocco. 2007. Historical and contemporary perspectives on mathematical learning disabilities. In Why Is Math So Hard for Some Children?, ed. D. B. Berch and M. M. M. Mazzocco. Brookes Publishing.

Griffin, S. A. 2007. Number Worlds: A Mathematics Intervention Program for Grades PreK-6. Columbus, OH: SRA/McGraw-Hill.

Gross, J., C. Hudson, and D. Price. 2009. The Long Term Costs of Numeracy Difficulties. Every Child a Chance Trust and KPMG.

Hanich, L. B., N. C. Jordan, D. Kaplan, and J. Dick. 2001. Performance across different areas of mathematical cognition in children with learning difficulties. Journal of Educational Psychology 93 (3): 615-626. http://dx.doi.org/10.1037//0022-0663.93.3.615

Hasselbring, T., R. Sherwood, J. Bransford, K. Fleenor, D. Griffith, and L. Goin. 1988. An evaluation of a level-one instructional videodisc program. Journal of Educational Technology Systems 16: 151-169. http://dx.doi.org/10.2190/BR31J510-CXM4-K41E

Hitch, G. J., and E. McAuley. 1991. Working memory in children with specific arithmetical learning difficulties. Br J of Psychol 82 (3): 375-386. http://dx.doi.org/10.1111/j.2044-8295.1991.tb02406.x 
Jordan, N. C., and L. B. Hanich. 2003. Characteristics of children with moderate mathematics deficiencies: A longitudinal perspective. Learning Disabilities: Research and Practice 18 (4) (November): 213-221. http://dx.doi.org/10.1111/1540-5826.00076

Koontz, K. L., and D. B. Berch. 1996. Identifying simple numerical stimuli: Processing inefficiencies exhibited by arithmetic learning disabled children. Mathematical Cognition 2 (1) (April 1): 1-24. http://dx.doi.org/10.1080/135467996387525

Kosc, L. 1970. Psychology and psychopathology of mathematical abilities. Studia Psychological 12: 159-162.

Kroeger, L. A., R. D. Brown, and B. A. O'Brien. 2012. Connecting neuroscience, cognitive, and educational theories and research to practice: A review of mathematics intervention program. Early Education \& Development 23 (1) (January): 37-58. http://dx.doi.org/10.1080/10409289.2012.617289

Landerl, K., A. Bevan, and B. Butterworth. 2004. Developmental dyscalculia and basic numerical capacities $\square$ : A study of 8-9-year-old students. Cognition 93: 99-125. http://dx.doi.org/10.1016/j.cognition.2003.11.004

Lindsay, R. L., T. Tomazic, M. D. Levine, and P. J. Accardo. 2001. Attentional function as measured by a continuous performance task in children with dyscalculia. $J$ Dev Behav Pediatr 22 (5): 287-292. http://dx.doi.org/10.1097/00004703-200110000$\underline{00002}$

Mazzocco, M. M. M., and G. F. Myers. 2003. Complexities in identifying and defining mathematics learning disability in the primary school-age years. Annals of Dyslexia 53 (1) (January 1): 218-253. http://dx.doi.org/10.1007/s11881-003-0011-7.

Mazzocco, M. M. M., K. T. Devlin, and S. J. McKenney. 2008. Is it a fact? Timed arithmetic performance of children with mathematical learning disabilities (MLD) varies as a function of how MLD is defined. Developmental Neuropsychology 33 (3) (January): 318-44. http://dx.doi.org/10.1080/87565640801982403

Mazzocco, M. M. M., L. Feigenson, and J. Halberda. 2011. Impaired acuity of the approximate number system underlies mathematical learning disability (dyscalculia). Child Development 82 (4) (July): 1224-37. http://dx.doi.org/10.1111/j.1467-8624.2011.01608.x

McLean, J. F., and G. J. Hitch. 1999. Working memory impairments in children with specific arithmetic learning difficulties. J Exp Child Psychol 74 (3): 240-260. http://dx.doi.org/10.1006/jecp.1999.2516

Moyer, R. S., and T. K. Landauer. 1967. Time required for judgements of numerical inequality. Nature 215 (109): 1519-1520. http://dx.doi.org/10.1038/2151519a0

Mussolin, C., A. De Volder, C. Grandin, X. Schlogel, M. C. Nassogne, and M. P. Noel. 2010. Neural correlates of symbolic number comparison in developmental dyscalculia. Journal of Cognitive Neuroscience. . 22 (5): 860-874. http://dx.doi.org/10.1162/jocn.2009.21237

Mussolin, C., S. Mejias, and M P Noel. 2010. Symbolic and nonsymbolic number comparison in children with and withoutdDyscalculia. Cognition 115 (1) (April): 10-25. http://dx.doi.org/10.1016/j.cognition.2009.10.006 
OECD. 2010. The High Cost of Low Education Performance: The Long-Run Economic Impact of Improving Educational Outcomes. Paris.

Parsons, S., and J. Bynner. 2005. Does Numeracy Matter More. NRDC (National Research and Development Centre for Adult Literacy and Numeracy), Institute of Education, London. http://eprints.ioe.ac.uk/4758/1/parsons2006does.pdf

Passolunghi, M. C., and L. S. Siegel. 2004. Working memory and access to numerical information in children with disability in mathematics. J Exper Child Psychol 88 (4) (August): 348-367. http://dx.doi.org/10.1016/j.jecp.2004.04.002

Pellegrino, J. W., and S. R. Goldman. 1987. Information processing and elementary mathematics. J Learn Disabil 20: 23-32. http://dx.doi.org/10.1177/002221948702000105

Piazza, M., A. Facoetti, A. N. Trussardi, I. Berteletti, S. Conte, D. Lucangeli, S. Dehaene, and M. Zorzi. 2010. Developmental trajectory of number acuity reveals a severe impairment in developmental dyscalculia. Cognition 116 (1) (July): 33-41. http://dx.doi.org/10.1016/j.cognition.2010.03.012

Price, G. R., I. D. Holloway, P. Räsänen, M. Vesterinen, and D. Ansari. 2007. Impaired parietal magnitude processing in developmental dyscalculia. Current Biology 17 (24) (December 18): 1042-1043. http://dx.doi.org/10.1016/j.cub.2007.10.013

Rotzer, S., K. Kucian, E. Martin, M. von Aster, P. Klaver, and T. Loenneker. 2007. Optimized voxel-based morphometry in children with developmental dyscalculia. Neuroimage 39 (1) (January 1): 417-422. doi:10.1016/j.neuroimage.2007.08.045.

Rotzer, S., T. Loenneker, K. Kucian, E. Martin, P. Klaver, and M. von Aster. 2009. Dysfunctional neural network of spatial working memory contributes to developmental dyscalculia. Neuropsychologia 47 (13) (November): 2859-65. http://dx.doi.org/10.1016/j.neuropsychologia.2009.06.009

Rousselle, L., and M. P. Noel. 2007. Basic numerical skills in children with mathematics learning disabilities: A comparison of symbolic vs. non-symbolic number magnitude processing. Cognition 102 (3): 361-395. http://dx.doi.org/10.1016/j.cognition.2006.01.005

Rubinsten, O., and A. Henik. 2005. Automatic activation of internal magnitudes: A study of developmental dyscalculia. Neuropsychology 19 (5) (September): 641-648. http://dx.doi.org/10.1037/0894-4105.19.5.641

. 2009. Developmental dyscalculia: Heterogeneity might not mean different mechanisms. Trends in Cognitive Sciences 13 (2) (February): 92-99. http://dx.doi.org/10.1016/j.tics.2008.11.002

Russell, R. L., and H. P. Ginsburg. 1984. Cognitive analysis of children's mathematical difficulties. Cognition \& Instruction 1: 217-244. http://dx.doi.org/10.1207/s1532690xci0102_3

Rykhlevskaia, E., L. Q. Uddin, L. Kondos, and V. Menon. 2009. Neuroanatomical correlates of developmental dyscalculia: Combined evidence from morphometry and tractography. Frontiers in Human Neuroscience 3 (November) (January): 51. http://dx.doi.org/10.3389/neuro.09.051.2009 
Räsänen, P., J. Salminen, A. J. Wilson, P. Aunio, and S. Dehaene. 2009. Computerassisted intervention for children with low numeracy skills. Cognitive Development 24 (4) (January): 450-472. http://dx.doi.org/10.1016/j.cogdev.2009.09.003

Sekuler, R., and D. Mierkiewicz. 1977. Children's judgments of numerical inequality. Child Development 48: 630-633. http://dx.doi.org/10.2307/1128664

Shalev, R. S., J. Auerbach, and V. Gross-Tsur. 1995. Developmental dyscalculia behavioral and attentional aspects: A research note. J Child Psychol Psychiatry 36 (7): 1261-1268. http://dx.doi.org/10.1111/j.1469-7610.1995.tb01369.x

Shalev, R. S., J. Auerbach, O. Manor, and V. Gross-Tsur. 2000. Developmental dyscalculia: Prevalence and prognosis. Eur Child Adolesc Psychiatry 9 Suppl 2 (Dc) (January): II58-64.

Shalev, R. S., O. Manor, and V. Gross-Tsur. 1997. Neuropsychological aspects of developmental dyscalculia. Mathematical Cognition 3 (2): 102-120. http://dx.doi.org/10.1080/135467997387434

Temple, C. M., and S. Sherwood. 2002. Representation and retrieval of arithmetical facts: Developmental difficulties. Quarterly Journal of Experimental Psychology 55A (3): 733-752.

van der Sluis, S, P. F de Jong, and A. van der Leij, 2004. Inhibition and shifting in children with learning deficits in arithmetic and reading. Journal of Experimental Child Psychology 87 (3): 239-266. http://dx.doi.org/10.1016/j.jecp.2003.12.002

Wilson, A. J, S. K. Revkin, and D. Cohen. 2006. An open trial assessment of "The Number Race", an adaptive computer game for remediation of dyscalculia. Behavioral and Brain Functions 2006, 2;20. http://dx.doi.org/10.1186/1744-9081-2$\underline{20}$ 